\title{
Use of Mesenchymal Stem Cells to Grow Cartilage and Bone
}

\author{
Hosseyn Rafei \\ Department of Biology, Faculty of Sciences, Islamic Azad University of Shiraz, Shiraz, Iran \\ Email: hosseyn.rafiei1@gmail.com
}

Received 11 May 2015; accepted 19 June 2015; published 23 June 2015

Copyright (C) 2015 by author and Scientific Research Publishing Inc. This work is licensed under the Creative Commons Attribution International License (CC BY). http://creativecommons.org/licenses/by/4.0/

c) (†) Open Access

\begin{abstract}
Regardless of the fact that bones have a tremendous capacity to be repaired, there are various clinical situations where allographs seek to enhance the regeneration of bones through promotion of osteogenesis [1]. Precarious botches linked to the present grafting treatments include osteonecrosis along with restricted combination between the grafted and host tissues. It is considered that the fundamental issue with the present approaches to bone grafting is the fact that they lead to bone regeneration by means of direct osteogenesis. Therefore, it may be hypothesized that the use of cartilage in the promotion of endochondral regeneration of bones may leverage normal development and repair sequences resulting in a properly vascularized regenerate capable of integrating with the host tissues. Various tests have demonstrated that cartilage grafts are able to support the regeneration of vascularized and integrated bone tissues in vivo, while lineage tracing experiments have revealed graft derived regenerates.
\end{abstract}

\section{Keywords}

Mesenchymal Stem Cells, Cartilage, Bone, Embryonic Sources

\section{Introduction}

Mesenchymal stem cells are actually the pluripotent cells existing in numerous adult human tissues, for example bone marrow and adipose as well as synovial tissues, and since they originate from the mesoderm, they differentiate to become bones and cartilage as well as muscles and adipose tissues [2]. Mesenchymal stem cells from embryonic sources have demonstrated probabilities in science while at the same time creating a considerable amount of controversy. As a consequence, numerous researchers have put emphasis on adult stem cells or those isolated from human adults that may be transplanted in to tissues that are damaged. As a result of their multi-potent capability, mesenchymal stem cell lineages have been employed with success in animal models in the 
regeneration of articular cartilage and in human models in the regeneration of bones. Research done recently has shown that articular cartilage can be repaired through percutaneous introduction of mesenchymal stem cells. This paper aims at explaining how bone and cartilage can be differentiated from stem cells as well as considering the current research and development of this discipline. This method is considered as an effective treatment option but is associated with some limitations, including donor-site morbidity, tissue supply limitation, unsuitable mechanical properties and thickness of the obtained tissue (Figure 1).

\section{Methods and Materials}

An experiment that sought to investigate conditions that can stimulate stem cells to differentiate into fat of bone cells while being encapsulated by hydrogels was conducted with polymer networks, which could simulate various conditions where the stem cells grow naturally. The first step in growing the new cartilage involved initiating chondrogenesis, which entails catalyzing the mesenchymal stem cells to differentiate themselves to become chondrocytes, which consequently develop a spongy matrix of collagen along with sugars that act as cushions for damaged joints. One of the challenges associated with catalyzing these cells to differentiate is that, regardless of the adult chondrocytes being of low density, the growth of cartilage starts when the cells are close to one another. In the hydrogels that are normally used form engineering cartilage tissue, cells are spaced apart and this makes them lose their signals and interactions. This is where the cadherins come in as they are the molecules that are utilized by the cells for interaction with each other especially at the point where they initially become chondrocytes. In order to simulate this environment, a peptide sequence that imitates the cadherin interactions had to be utilized and they bound to the hydrogels used in order to encapsulate the stem cells (The collagen hydrogel used for matrix-based MSC transplantation was fabricated from rat-tail collagen).

Even though the direct connection between cadherins and chondrogenesis has not been entirely understood, it is clear that the interactions are enhanced in the process of tissue formation, more cartilage can be made, but if they are blocked, then the cartilage that will be formed will be poor. So that the efficiency of the peptide that mimics cadherins could be tested, the mesenchymal stem cells were encapsulated in several other types of gels including hydrogel that lacked peptide, hydrogel containing non-functional peptide and one that contained both peptide and antibodies blocking the interaction of cadherins. In a week's time, the cells in the gel that contained cadherin peptide demonstrated increased genetic markers in terms of chondrogenesis compared to the cells that were placed in the other controls.

\section{Results}

The capability of mesenchymal stem cells to differentiate into bone cells referred to as osteoblasts has resulted in their usage in clinic experiments that investigate the safety of probable methods of repairing bones. The trials focus on potential treatments for skeletal defects of a localized nature such as damage at a specific position in
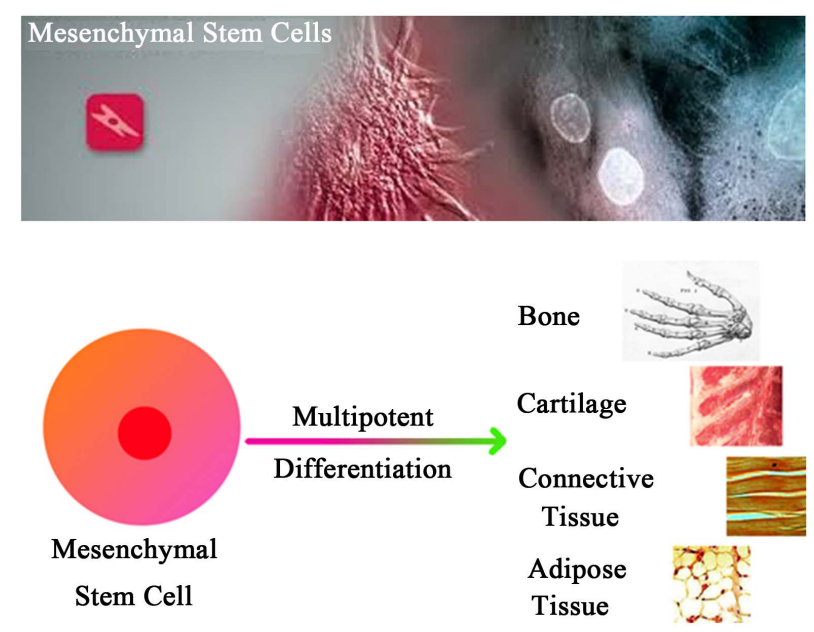

Figure 1. Mesenchymal stem cell differentiate to become bones and cartilage as well as muscles and adipose tissues. 
the bone. Additional research focuses on the use of mesenchymal stem cells in repairing cartilage, which covers the bone ends and makes it possible for one bone to slide over another one where they are joined. A cartilage may be damaged through injuries such as falls or over long periods through conditions such as osteoarthritis, which is an extremely painful disease that affects the joints.

Cartilage does not have an ability to repair itself completely after it is damaged and the best treatment for acute damage to the cartilage is surgery that replaces the joint that has been damaged artificially [3]. Since the mesenchymal stem cells have an ability to differentiate into cells that form the cartilage known as chondrocytes, researchers are hopeful that they could be injected into people with damaged cartilages in order to repair and preserve cartilage. Scientists are also evaluating the probability that transplanted mesenchymal stem cells could release substances that will motivate the stem cells of the patient with damaged joints to mend the injury. Numerous obstacles remain and hinder this form of treatment from becoming a reality at present, for instance, when the mesenchymal stem cells are transplanted; huge majorities are quickly eliminated from the body. Scientists are developing newer approaches for transplanting these cells including the development of three-dimensional structures that imitate the body conditions that require these cells. These structures are supposed to hold the cells while encouraging differentiation in to the needed types of cells.

Additionally, marrow nucleated cells are utilized in regenerative orthopedics on a daily basis, where the knee micro-fracture surgery depends on releasing these cells into a cartilage lesion in order to start fibro-cartilage repairs in defects of an osteochondral nature. Furthermore, this type of cells has also been demonstrated to be useful in the repair on fractures of a non-union nature. In these cases, bedside centrifugation is usually employed but this approaches result in very dilute mesenchymal stem cell populations of around one in 10,000 to $1,000,000$ of cells that are nucleated. Notwithstanding this small number of mesenchymal stem cells, nucleated cells from bone marrow that has been isolated which are implanted into degenerated joints have demonstrated some level of promise as far as the repair of joints is concerned. Since the number of mesenchymal stem cells that may be isolated from bone marrow is relatively low, most of the research concerned with the regeneration of cartilage has been centered on usage of cells that have been expanded through being cultured [4]. This approach is able to expand the number of cells by between one hundred and ten thousand in a span of weeks and when the mesenchymal stem cells are ready to be re-implanted, they are typically transferred alongside growth factors in order to enable continued growth of cells as well as grafting of the tissues that had been damaged. Consequently, there is introduction of a signal, either during the culturing process or after the cells have been transplanted into the tissues that were damaged, that will enable the cells to differentiate into the tissues that are needed in the end.

Previously, cultured mesenchymal stem cells in the regeneration of cartilage was employed predominantly in research that concerned animal models but currently, there are two case reports that have been published of this approach being employed to regenerate articular and meniscus cartilages with success in knees of human beings. This approach has not yet been demonstrated as efficient in studies that involve a bigger group of patients; nonetheless, a large safety study has been published by the same researchers that has showed a smaller amount of complications than would typically be linked to surgical procedures. A different team employed the same approach while extracting cells as well as in ex vivo expansion but the cells had to set in collagen gel prior to being re-implanted surgically. This team reported a case study whereby a full-thickness defect that had affected the articular cartilage of a patient was repaired with success.

\section{Discussion}

\subsection{Regrowth of Broken Bones and Damaged Cartilages}

Damaged cartilages as well as broken bones may be fixed through simple operations that involve the injection of stem cells into the areas that have been damaged. Various researchers and scientists have identified the stem cells that may give rise to bones, cartilages as well as bone marrow and the manner in which they work. The scientists have also found the mechanisms that enable fat cells to differentiate to become skeleton stem cells and this discovery has offered hope for a newer treatment for skeletal disorders including fractures to the bone, brittle bones as well as injured or damaged cartilages. Numerous times every year, orthopedic surgeons come across damaged cartilages in joints and are obligated to take them out since cartilages are not able to heal themselves well, however, a lack of cartilage may cause the patient to eventually develop arthritis [5]. Therefore, the research associated with mesenchymal stem cells has increased the probability of creating new skeletal stem cells 
from the tissues of the affected patients so that they can be utilized in the growth of new cartilages.

Researchers are optimistic that this step forward will be valuable to the growth of missing body parts and cartilages in a laboratory after which they may be transplanted, as this will decrease the chances of them being rejected. Presently, if an individual losses a considerable part of the jawbone or leg, a different bone such as the fibula must be cut into the shape needed and then moved and attached to the blood supply. However, if the patient's existing bone is unavailable or insufficient, the current research might have a capacity to put some of the patient's fat into a biomimetic structure and allowed to grow into the bone that is needed in a muscle or fat pocket before it can be moved to the position where it is required. Researchers continue to be hopeful that they could catalyze fat cells to become skeleton stem cells that may be injected into the areas that are injured using a simple operation. This may be specifically useful and valuable in operations that involve knees and hips especially for elderly patients and the prevention of arthritis.

Since the amount of skeletal stem cells greatly reduces, as a person gets older, fractures of the bone and any forms of dental implants may not heal adequately in elderly patients, as new bones do not grow easily [6]. With the research in the skeletal stem cells, it will be possible to harvest fat from the body of the patient in the process of surgery in order to combine it with the reprograming factors and immediately transplant the created stem in to the patient who was undergoing the operation. Regardless of the fact that scientist have only mapped up to this point the skeletal stem cell structure on mice, they continue to be have confidence that they will have an ability to perform a similar procedure on human patients. This research has a considerable headway that should be valuable in finding human skeletal stem cells in order to understand the chemical aspects they employ to drive their development. Their application to human beings is considered to be similar and sharing the key genes that are utilized in the skeletal structures of mice.

\subsection{Applications of Mesenchymal Stem Cells}

The mesenchymal stem cells are among the tissues that originate from adult stem cells with multi-potent characteristics, meaning that they have a capacity to produce more than one form of specialized body cells [7]. These cells constitute the various specialized cells that are present in the skeletal tissues, for instance, they have an ability to differentiate into cartilage, bone or fat cells. Every mesenchymal cell has its unique characteristic shape, structure and function with each belonging in a specific tissue. Research has suggested that mesenchymal stem cells may also have a capacity to differentiate into numerous forms of cells that might not be a part of the skeletal tissues like nerve, heart muscle and liver cells that constitute the inner layer of the blood vessels. Nonetheless, these results have not yet been substantiated and in some of the cases, it seems like the mesenchymal stem cells fuse with the existing specialized cells resulting in a false conclusion concerning the capacity of the stem cells to produce particular types of cells. In different scenarios, the outcomes were artificial effects caused by chemicals that are utilized in growing the cells in the laboratory.

The mesenchymal stem cells originate from the bone marrow and there are claims that they can be found in various other tissues including blood from the umbilical code, adipose tissues as well as muscles. Full substantiation has not occurred concerning if the cells originating from the other tissues are actually the same as the mesenchymal stem cells originating from the bone marrow. In the bone marrow, there are numerous different forms of cells including red blood cells and a range of cells that belong to a group referred to as mesenchymal cells, however, only around 0.001 to 0.01 percent of bone marrow cells are actually mesenchymal stem cells [8]. Obtaining a blend of different mesenchymal cell forms for use in research is relatively easy in the case of adult bone marrow. However, isolation of the small fractions of cells, which are mesenchymal stem cells, is a more complex endeavor as some of the cells in the mixture may have an ability to develop into fat or bone tissues while not having all the characteristics associated with mesenchymal stem cells. The main challenge remains identifying and picking out the cells have an ability to renew themselves while at the same time differentiating into three types of cells including bone, fat and cartilage, but scientist have not yet been able to agree on the best way to achieve this. Consequently, no treatments that use mesenchymal stem cells that are available now, but a number of prospects for their clinical use that are presently under exploration.

\subsection{Continuing Research}

Research associated with mesenchymal stem cells has tremendously increased in the recent times with the most commonly used source of the stem cells being bone marrow aspirates. A large percentage of the adult bone 
marrow is comprised of blood cells that are in different stages of differentiation, which can be classified into plasma, platelets, red blood cells and nucleated cells. Factions of stem cells that are retrieved from adults exist in the marrow's cells that are nucleated. Majority of the cells are CD34+heme-originators, which will eventually differentiate into some of the components of blood, while a limited number have the capability to undergo differentiation into bones, cartilage or muscles. Based on this fact, the limited number of mesenchymal stem cells present in the marrow is the cells that have the capability to differentiate into tissues of interest as far as joint preservation is concerned [9]. It is imperative to note that this may be a reason why centrifuge systems that are available commercially concentrating marrow nucleated cells have not demonstrated significant promise in animal research concerned with the repair of cartilage when compared to the approaches where the mesenchymal stem cells undergo expansion in culture to increase their numbers.

\section{Conclusion}

The prevailing methods employed in regenerating bones and cartilage utilize adult stem cells with are valuable in the replacement and regeneration of tissues that may be affected by diseases or those that are damaged. Stem cells from human beings are differentiated into cells that work in the same manner as the embryonic stem cells, however, this approach has demonstrated to be costly with a low probability of being successful. The currents strategies used for the regeneration of cells generally utilize proteins for directing stems cells in order to produce functional cells that are needed. Additionally, proteins are relatively unstable and costly to produce and this constraint in the cost is among the obstacles that have restricted that capability of researchers to produce the amounts that are required for the clinical purposes. In order to circumvent this hurdle, researchers have to take a different approach that work on pluripotent cells that originate from early mouse embryos as they have an ability to transform to become any cell form. Researchers are considering the use of tiny molecules to catalyze the stem cells from the embryos so that they can differentiate into chondrocytes, which are the only cells that have an ability to form cartilages and eventually bones. The main reason why tiny molecules are used is that they usually last longer than proteins as far as culture is concerned and are less costly when produced in large-scales. These cells call also enable specific mechanisms to be activated in ways that are more accurate and this kind of approach has already been employed in the replacement of protein factors with the tiny molecules in order to develop a better culture approach in regard to maintaining pluripotent embryonic stem cells as well as inducing early precursor cells.

\section{References}

[1] Özkaya, N. and Özkaya, N. (2012) Fundamentals of Biomechanics. Springer, New York. http://dx.doi.org/10.1007/978-1-4614-1150-5

[2] Nolta, J. (2006) Genetic Engineering of Mesenchymal Stem Cells. Springer, Dordrecht. http://dx.doi.org/10.1007/1-4020-3959-X

[3] Cech, D. and Martin, S. (2012) Functional Movement Development across the Life Span. Saunders, St. Louis.

[4] Laughlin, M. and Lazarus, H. (2010) Allogeneic Stem Cell Transplantation. Humana Press, Totowa.

[5] Khurana, J. (2009) Bone Pathology. Humana Press, Dordrecht. http://dx.doi.org/10.1007/978-1-59745-347-9

[6] Dib, N., Taylor, D. and Diethrich, E. (2006) Stem Cell Therapy and Tissue Engineering for Cardiovascular Repair. Springer, New York. http://dx.doi.org/10.1007/0-387-30939-X

[7] Turksen, K. (2012) Adult and Embryonic Stem Cells. Humana Press, New York. http://dx.doi.org/10.1007/978-1-61779-630-2

[8] Sorensen, M. (2008) Stem Cell Applications in Diseases. Nova Biomedical Books, New York.

[9] Fisher, J. (2013) Tissue Engineering. CRC Press, Boca Raton. 